\title{
Monotonicity of quantum ground state energies: Bosonic atoms and stars
}

\author{
Michael K.-H. Kiessling \\ Department of Mathematics, Rutgers University \\ Piscataway NJ 08854, USA
}

\begin{abstract}
The $N$-dependence of the non-relativistic bosonic ground state energy $\mathcal{E}^{\mathscr{B}}(N)$ is studied for quantum $N$-body systems with either Coulomb or Newton interactions. The Coulomb systems are "bosonic atoms," with their nucleus fixed, and it is shown that $\mathcal{E}_{\mathscr{C}}^{\mathscr{B}}(N) / \mathcal{P}_{\mathscr{C}}(N)$ grows monotonically in $N>1$, where $\mathcal{P}_{\mathscr{C}}(N)=N^{2}(N-1)$. The Newton systems are "bosonic stars," and it is shown that when the Bosons are centrally attracted to a fixed gravitational "grain" of mass $M>$ 0 , and $N>2$, then $\mathcal{E}_{\mathscr{N}}^{\mathscr{B}}(N ; M) / \mathcal{P}_{\mathscr{N}}(N)$ grows monotonically in $N$, where $\mathcal{P}_{\mathscr{N}}(N)=N(N-1)(N-2)$; in the translation-invariant problem $(M=0)$, it is shown that when $N>1$ then $\mathcal{E}_{\mathscr{N}}^{\mathscr{B}}(N ; 0) / \mathcal{P}_{\mathscr{C}}(N)$ grows monotonically in $N$, with $\mathcal{P}_{\mathscr{C}}(N)$ from the Coulomb problem. Some applications of the new monotonicity results are discussed.
\end{abstract}

Typeset in LATEX by the author. Revised version of Sept. 10, 2009.

(C)2009 The author. This preprint may be reproduced for noncommercial purposes. 


\section{Introduction}

While bosonic matter in bulk has been a subject of intense theoretical research over the years, spurned on in particular by the recent breakthroughs in creating Bose-Einstein condensates in the laboratory, theoretical research into the properties of individual bosonic atoms could seem to always remain of purely academic interest, for there are no bosonic electrons in nature. Yet in principle bosonic atoms can exist in nature as we know it, and not just in the "artificial" sense described in [KSK04]. Namely, they can be formed with $N$ bosonic anti- $\alpha$ particles of charge $-2 e$ and spin 0 each playing the rôle of bosonic electrons which are attracted by a nucleus of charge $2 e N$, conceivably up to $N \approx 46$. Both varieties of particles would have to be produced in a laboratory, the nucleus by "just" stripping away all electrons from its associated atom, unless the nucleus is itself an $\alpha$ particle which nature supplies through some radioactive materials. This latter case yields the simplest (i.e. $N=1$ ) $\alpha$-bosonic atom, "alphium," the $\alpha$ particle analog of protonium 1 and of the familiar positronium (which would have better been called "electronium"). The $N=2 \alpha$-bosonic atom would have a Beryllium nucleus, the stable isotope of which $\left({ }^{9} \mathrm{Be}\right)$ is a fermion with spin $3 / 2$; and so on. Since fermionic anti- ${ }^{3} \mathrm{He}$ nuclei have already been produced in heavy ion collisions at CERN Aetal96, Aetal03a, Aetal03b], it seems a safe bet to predict that also bosonic anti- $\alpha$ particles are going to be produced in the laboratory 2 and that research into individual bosonic atoms will take off once they can be captured in large enough numbers and made to form bound states with a normal nucleus 3

Like positronium and protonium, both of which have lifespans of the order of $\mu$-seconds, also alphium and the heavier $\alpha$-bosonic atoms will be shortlived, but since the involved $\alpha$ and anti- $\alpha$ particles are compounds of protons and neutrons, respectively their anti-particles, which according to the standard model are themself bound states of up and down quarks, respectively anti-quarks, the annihilation modes will be more complicated - and more interesting - than those of protonium 4 and vastly more so than those of positronium 5 Thus bosonic atoms would also open up a new window for studying the strong interactions at lower energies.

\footnotetext{
${ }^{1}$ Protonium, which is a fermionic atom made of a proton and an anti-proton, recently became an experimental reality [Zetal06a, Zetal06b].

${ }^{2}$ Should the production of even heavier anti-nuclei become feasible some day, then one could also enlist $N>1$ bosonic $\alpha$ particles of charge $2 e$ and spin 0 for playing the rôle of bosonic electrons orbiting an anti-nucleus of charge $-2 e N$. Even heavier bosonic atoms are conceivable, e.g. with $N$ Neon nuclei ${ }^{20} \mathrm{Ne}$ of charge $10 e$ and spin 0 each attracted by an anti-nucleus of charge $-10 e N$, perhaps up to $N \approx 9$.

${ }^{3}$ It would be foolish, though, to predict when this will become an experimental reality.

${ }^{4}$ For an early attempt at calculating effective decay channels of protonium, see Des60.

${ }^{5}$ The vacuum decay channels of positronium are well-known, though not yet completely understood CzKa00.
} 
In the meantime theoretical research into the properties of bosonic atoms remains the only available venue of inquiry. Following widespread custom [BeLi83], [Lie90], [Sol90], Bac91], BLLS93], Rus97], [BaSe01], [Thi02], in this paper we study the simplified non-relativistic problem with the atomic nucleus fixed at the origin of a co-ordinate system. We will show that $\mathcal{E}_{\mathscr{C}}^{\mathscr{B}}(N) / \mathcal{P}_{\mathscr{C}}(N)$ grows monotonically in $N>1$, where $\mathcal{E}_{\mathscr{C}}^{\mathscr{B}}(N)$ is the bosonic ground state energy of the fixed-nucleus atomic Coulomb Hamiltonian, and $\mathcal{P}_{\mathscr{C}}(N)=N^{2}(N-$ 1). The Galilei-invariant atomic model with a dynamical nucleus is a more tricky $N+1$-body problem which we hope to address in the future.

Our technique of proving monotonicity of the bosonic ground state energy for the atomic Coulomb system easily handles also some gravitational Newton system modeling a non-relativistic "bosonic star." While we have argued that bosonic atoms can in principle exist in nature as we know it and that we expect them to be produced in laboratories eventually, it is not clear to the author whether bosonic stars will ever be more than theoretical speculation. In any event, theoretical studies of the bosonic ground state energy for $N$ body Schrödinger operators with gravitational Newton interactions have a long tradition, see [Pos62], [L-L69], Hal88, Hal92], BMR90], Thi02] (see also [LiYa87, Lie90], HaLu06, HaLu08], [FrLe09] for some semi-relativistic models), and so we may as well contribute to it.

We will first show that when the Bosons are centrally attracted to a fixed gravitational "grain" of mass $M>0$, and $N>2$, then $\mathcal{E}_{\mathscr{N}}^{\mathscr{B}}(N ; M) / \mathcal{P}_{\mathscr{N}}(N)$ is finite and grows monotonically in $N$, where $\mathcal{P}_{\mathscr{N}}(N)=N(N-1)(N-2)$. Fixing some attracting center — the nucleus in the atomic and a "grain" in the stellar case - is a convenient technical ruse which ensures the existence of a ground state and simplifies the mathematics. However, while a fixed nucleus is a physically justifiable approximation for normal atoms because of their large nucleon-to-electron mass ratio, and marginally acceptable for bosonic atoms made of anti- $\alpha$ particles bound to a sufficiently large normal nucleus, fixing a gravitational grain is entirely artificial and, to the best of the author's knowledge, has not yet attracted much attention in the mathematical physics community. The problem with a fixed attracting center is of interest chiefly because the limit $\lim _{M \downarrow 0} \mathcal{E}_{\mathscr{N}}^{\mathscr{B}}(N ; M) \equiv \mathcal{E}_{\mathscr{N}}^{\mathscr{B}}\left(N ; 0^{+}\right)$is not only a lower bound for the actual $M=0$ "ground state" energy (now read: infimum) $\mathcal{E}_{\mathscr{N}}^{\mathscr{B}}(N ; 0)$ of the proper Galilei-invariant $N$-body operator of a bosonic star (obtained by simply setting $M=0$ in the operator with fixed attracting center of mass $M$ ), it can in fact be shown ReSi78] that $\mathcal{E}_{\mathscr{N}}^{\mathscr{B}}\left(N ; 0^{+}\right)$equals $\mathcal{E}_{\mathscr{N}}^{\mathscr{B}}(N ; 0)$. And so, since $\mathcal{P}_{\mathscr{N}}(N)$ is independent of $M$, we conclude that also $\mathcal{E}_{\mathscr{N}}^{\mathscr{B}}(N ; 0) / \mathcal{P}_{\mathscr{N}}(N)$ is finite for $N \geq 3$ and grows monotonically in $N$.

Interestingly enough, though, we get a stronger monotonicity result for $\mathcal{E}_{\mathscr{N}}^{\mathscr{B}}(N ; 0)$ by applying our technique directly to the $M=0$ Galilei-invariant $N$-body operator. Namely, the familiar reduction of the two-body Hydro- 
gen problem to an effective one-body problem with attractive center makes it plain that the translation-invariant $N$-body problem is effectively an $N-1$ body problem with attractive center in disguise, obtained by subtracting the energy for the degrees of freedom of the $N$-body system's center-of-mass off from the Hamiltonian without changing the value of the "inf" (though rendering a "min" for the so-obtained "intrinsic Hamiltonian"). Our technique applied directly to the reduced Galilei-invariant $M=0$ problem, i.e. the intrinsic Hamiltonian, produces the stronger monotonicity law that, when $N>1$ then $\mathcal{E}_{\mathscr{N}}^{\mathscr{B}}(N ; 0) / \mathcal{P}_{\mathscr{C}}(N)$ is finite and grows monotonically in $N$, with $\mathcal{P}_{\mathscr{C}}(N)$ as before. Note that this monotonicity law implies the monotonicity of $\mathcal{E}_{\mathscr{N}}^{\mathscr{B}}(N ; 0) / \mathcal{P}_{\mathscr{N}}(N)$ for $N \geq 3$.

The precise statements of our results are given in section II, their proofs in section III. Section IV recalls the Hall-Post inequalities and shows that these are further spin-offs of our techniques. We conclude our paper in section $\mathrm{V}$ with an outlook on the fermionic ground state energies.

\section{Results}

\subsection{Bosonic atoms with a fixed nucleus}

Whether one takes $N$ bosonic anti-nuclei of charge - ze each, which repell each other by Coulomb's law and are attracted to a nucleus of charge $N z e$ by its electrical Coulomb field, or $N$ bosonic charges ze in the field of an anti-nucleus of charge $-N z e$, with $z \in \mathbb{N}$, when the (anti-)nucleus is fixed at the origin the non-relativistic $N$-body Hamiltonian for such a bosonic atom in either case is given by the formal Schrödinger operator

$$
H_{\mathscr{C}}^{(N)} \equiv \sum_{1 \leq k \leq N}\left(\frac{1}{2 m}\left|\boldsymbol{p}_{k}\right|^{2}-N z^{2} e^{2} \frac{1}{\left|\boldsymbol{q}_{k}\right|}\right)+\sum_{1 \leq j<k \leq N} \sum_{z^{2}} e^{2} \frac{1}{\left|\boldsymbol{q}_{k}-\boldsymbol{q}_{j}\right|}
$$

where the subscript $\mathscr{C}$ indicates the electrical Coulomb interactions, and $m$ is the Newtonian inertial mass of each of the $N$ particles. In (1), $\boldsymbol{p}_{k}=-i \hbar \nabla_{k}$ is the familiar momentum operator canonically dual to the $k$-th component of the configuration space position operator $\boldsymbol{q}_{k} \in \mathbb{R}^{3}$. The formal operator $H_{\mathscr{C}}^{(N)}$ is densely defined on $\mathfrak{C}_{0}^{\infty}\left(\mathbb{R}^{3}\right) \cap \mathfrak{L}^{2}\left(\mathbb{R}^{3 N}\right)$.

As self-adjoint extension we take its Friedrichs extension, also denoted by $H_{\mathscr{C}}^{(N)}$, which is a permutation-symmetric, self-adjoint operator with form domain given by the $N$-fold tensor product $D_{Q}^{(N)} \equiv \mathfrak{H}^{1}\left(\mathbb{R}^{3}\right) \otimes \cdots \otimes \mathfrak{H}^{1}\left(\mathbb{R}^{3}\right) \subset$ $\mathfrak{L}^{2}\left(\mathbb{R}^{3 N}\right)$. The quadratic form associated to the operator $H_{\mathscr{C}}^{(N)}$ is

$$
Q_{\mathscr{C}}^{(N)}\left(\psi^{(N)}\right)=\frac{\hbar^{2}}{2 m} \mathcal{K}^{(N)}\left(\psi^{(N)}\right)-N z^{2} e^{2} C^{(N)}\left(\psi^{(N)}\right)+z^{2} e^{2} I^{(N)}\left(\psi^{(N)}\right),
$$


where (with integrals extending over $\mathbb{R}^{3 N}$ )

$$
\begin{aligned}
\mathcal{K}^{(N)}\left(\psi^{(N)}\right) & =\int \sum_{1 \leq k \leq N}\left|\nabla_{k} \psi^{(N)}\right|^{2} d^{3 N} q, \\
\mathcal{C}^{(N)}\left(\psi^{(N)}\right) & =\int \sum_{1 \leq k \leq N} \frac{1}{\left|\boldsymbol{q}_{k}\right|}\left|\psi^{(N)}\right|^{2} d^{3 N} q, \\
I^{(N)}\left(\psi^{(N)}\right) & =\int \sum_{1 \leq k<l \leq N} \sum_{1 \leq N} \frac{1}{\left|\boldsymbol{q}_{k}-\boldsymbol{q}_{l}\right|}\left|\psi^{(N)}\right|^{2} d^{3 N} q .
\end{aligned}
$$

The bosonic ground state energy of $H_{\mathscr{C}}^{(N)}$ is defined by

$$
\mathcal{E}_{\mathscr{C}}^{\mathscr{B}}(N) \equiv \min \left\{Q_{\mathscr{C}}^{(N)}\left(\psi^{(N)}\right) \mid \psi^{(N)} \in D_{Q}^{(N)} ;\left\|\psi^{(N)}\right\|_{\mathfrak{L}^{2}\left(\mathbb{R}^{3 N}\right)}=1\right\}
$$

It is well known, e.g. [Thi02, that a minimizing ground state $\psi_{\mathscr{B}}^{(N)}$ for (6) exists, and that by the permutation symmetry of $H_{\mathscr{C}}^{(N)}$ the minimizer is permutation symmetric, too, hence "bosonic." The variational problem (6) has been studied in [BeLi83], [Lie90], [Sol90], Bac91], BLLS93], Rus97], BaSe01], Thi02]; yet the following monotonicity result for $\mathcal{E}_{\mathscr{C}}^{\mathscr{B}}(N)$ seems new.

Proposition 1. Let $\mathcal{E}_{\mathscr{C}}^{\mathscr{B}}(N)$ denote the bosonic ground state energies defined in ([6), and let $\mathcal{P}_{\mathscr{C}}(N)=N^{2}(N-1)$. Then for $N \geq 2$ the ratio $\mathcal{E}_{\mathscr{C}}^{\mathscr{B}}(N) / \mathcal{P}_{\mathscr{C}}(N)$ is finite and grows monotonically in $N$.

Proposition 1 has some interesting spin-offs.

Corollary 1. For $N>1$ we have

$$
\mathcal{E}_{\mathscr{C}}^{\mathscr{B}}(N) \geq \mathcal{E}_{\mathscr{C}}^{\mathscr{B}}(2) \frac{1}{4} N^{3}\left(1-N^{-1}\right)
$$

The lower bound (17) on $\mathcal{E}_{\mathscr{C}}^{\mathscr{B}}(N)$ is sharp for $N=2$, but certainly not optimal for large $N$. The Helium-type ground state energy $\mathcal{E}_{\mathscr{C}}^{\mathscr{B}}(2)$ can be calculated, not exactly, but approximately with high precision using the method of Hylleraas [Hyl30. The bound (7) may be compared with the bound obtained by setting $k=1$ and $z_{1}=N$ in formula (6.1) in [Lie90, which reads

$$
\left.\mathcal{E}_{\mathscr{C}}^{\mathscr{B}}(N) \geq- \text { const. }\right) N^{3}\left(1+N^{-4 / 3}\right) .
$$

Both bounds have the same leading order power in $N$; if "(const.)" in (8) is $\geq-\frac{1}{4} \mathcal{E}_{\mathscr{C}}^{\mathscr{B}}(2)$, then (7) improves over (8) for all $N$, but if "(const.)" $<-\frac{1}{4} \mathcal{E}_{\mathscr{C}}^{\mathscr{B}}(2)$, then (18) beats (77) for $N>N_{*}$, with $N_{*}$ depending on "(const.)." Of course, since (7) is sharp for $N=2$, "(const.)" cannot be smaller than $-\mathcal{E}_{\mathscr{C}}^{\mathscr{B}}(2) /(8+$ $\left.2^{5 / 3}\right)$, and if "(const.)" equals this value then (8) beats (7) for all $N>2$. 
To state our second spin-off of Proposition 1 we recall that an upper bound to the bosonic ground state energy $\mathcal{E}_{\mathscr{C}}^{\mathscr{B}}(N)$ is obtained by estimating $\mathcal{Q}_{\mathscr{C}}^{(N)}\left(\psi^{(N)}\right)$ from above with the help of a convenient trial wave function $\psi^{(N)} \equiv \phi^{\otimes N} \in$ $D_{Q}^{(N)}$, with $\phi \in \mathfrak{H}^{1}\left(\mathbb{R}^{3}\right)$. We have $Q_{\mathscr{C}}^{(N)}\left(\phi^{\otimes N}\right)=\mathcal{H}_{\mathscr{C}}^{(N)}(\phi)$, with

$$
\mathcal{H}_{\mathscr{C}}^{(N)}(\phi)=N \frac{\hbar^{2}}{2 m} \mathcal{K}^{(1)}(\phi)-N^{2} z^{2} e^{2} C^{(1)}(\phi)+N(N-1) z^{2} e^{2} \frac{1}{2} I^{(2)}(\phi \otimes \phi)
$$

a Hartree functional. Setting $\phi(\boldsymbol{q})=N^{3 / 2} \phi_{0}(N \boldsymbol{q})$ yields the well-known upper bound $\mathcal{E}_{\mathscr{C}}^{\mathscr{B}}(N) \leq-C_{0} N^{3}$. Pairing it with Proposition 1 we conclude

Corollary 2. The limit $\lim _{N \uparrow \infty} N^{-3} \mathcal{E}_{\mathscr{C}}^{\mathscr{B}}(N)$ exists and is non-trivial.

Our arguments do not reveal the nature of such a limit. In [BeLi83] it is shown that the limit is given by the minimum of the Hartree functional

$$
\mathcal{H}_{\mathscr{C}}(\phi)=\frac{\hbar^{2}}{2 m} \mathcal{K}^{(1)}(\phi)-z^{2} e^{2} C^{(1)}(\phi)+z^{2} e^{2} \frac{1}{2} I^{(2)}(\phi \otimes \phi)
$$

over normalized $\mathfrak{H}^{1}\left(\mathbb{R}^{3}\right)$; see also [Lie90], [Sol90], [Bac91], [BaSe01], [Thi02].

\subsection{Bosonic stars with a fixed gravitational center}

The formal Schrödinger operator for a bosonic star with fixed gravitational center reads

$$
H_{\mathscr{N}, M}^{(N)} \equiv \sum_{1 \leq k \leq N}\left(\frac{1}{2 m}\left|\boldsymbol{p}_{k}\right|^{2}-G M m \frac{1}{\left|\boldsymbol{q}_{k}\right|}\right)-\sum_{1 \leq j<k \leq N} G m^{2} \frac{1}{\left|\boldsymbol{q}_{j}-\boldsymbol{q}_{k}\right|},
$$

with $M>0$. The operators of the parameter $(M)$ family (11) are densely defined and symmetric on $\mathfrak{C}_{0}^{\infty}\left(\mathbb{R}^{3}\right) \cap \mathfrak{L}^{2}\left(\mathbb{R}^{3 N}\right)$, and as self-adjoint extension of (11) we again take its Friedrichs extension, also denoted by $H_{\mathscr{N}, M}^{(N)}$, a permutationsymmetric, self-adjoint operator with form domain given by the $N$-fold tensor product $D_{Q}^{(N)} \equiv \mathfrak{H}^{1}\left(\mathbb{R}^{3}\right) \otimes \cdots \otimes \mathfrak{H}^{1}\left(\mathbb{R}^{3}\right) \subset \mathfrak{L}^{2}\left(\mathbb{R}^{3 N}\right)$. The quadratic form associated to the operator $H_{\mathscr{N}, M}^{(N)}$ is

$$
Q_{V, M}^{(N)}\left(\psi^{(N)}\right)=\frac{\hbar^{2}}{2 m} \mathcal{K}^{(N)}\left(\psi^{(N)}\right)-G M m C^{(N)}\left(\psi^{(N)}\right)-G m^{2} I^{(N)}\left(\psi^{(N)}\right),
$$

where $\mathcal{K}^{(N)}, C^{(N)}$, and $I^{(N)}$ are defined in (3), (44), and (5), respectively. The bosonic ground state energy of $H_{\mathscr{N}, M}^{(N)}$ for $M>0$ is defined by

$$
\mathcal{E}_{\mathscr{N}}^{\mathscr{B}}(N ; M) \equiv \min \left\{Q_{N, M}^{(N)}\left(\psi^{(N)}\right) \mid \psi^{(N)} \in D_{Q}^{(N)} ;\left\|\psi^{(N)}\right\|_{\mathfrak{L}^{2}\left(\mathbb{R}^{3 N}\right)}=1\right\} .
$$

By the permutation symmetry of $H_{\mathscr{N}, M}^{(N)}$, the minimizer for (13) with $M>0$, denoted $\psi_{\mathscr{B}, M}^{(N)}$, is permutation symmetric, too, hence "bosonic."

We will show that the bosonic ground state energies $\mathcal{E}_{\mathscr{N}}^{\mathscr{B}}(N ; M)$ exhibit a monotonic dependence on $N$ similar to Proposition 11. 
Proposition 2. For $M>0$ let $\mathcal{E}_{N}^{\mathscr{B}}(N ; M)$ denote the bosonic ground state energies defined in (13), and let $\mathcal{P}_{\mathscr{N}}(N)=N(N-1)(N-2)$. Then for $N \geq 3$ the ratio $\mathcal{E}_{\mathscr{N}}^{\mathscr{B}}(N ; M) / \mathcal{P}_{\mathscr{N}}(N)$ is finite and grows monotonically in $N$.

Also Proposition 2 has two technical spin-offs. The first one is immediate:

Corollary 3. For $N>2$ we have

$$
\mathcal{E}_{\mathscr{N}}^{\mathscr{B}}(N ; M) \geq \mathcal{E}_{\mathscr{N}}^{\mathscr{B}}(3 ; M) \frac{1}{6} N^{3}\left(1-N^{-1}\right)\left(1-2 N^{-1}\right) .
$$

The lower bound (14) on $\mathcal{E}_{\mathscr{N}}^{\mathscr{B}}(N ; M)$ is sharp for $N=3$, but far from sharp when $N \gg 1$. The coefficient $\mathcal{E}_{N}^{\mathscr{B}}(3 ; M)$ can be estimated from below 6 uniformly in $M$, in terms of the two-body ground state energy with central mass $M / 2$ and gravitational constant $2 G$, as follows: $\mathcal{E}_{\mathscr{N}}^{\mathscr{B}}(3 ; M) \geq 3 \mathcal{E}_{2 \mathscr{N}}^{\mathscr{B}}(2 ; M / 2)$, where the notation " $2 \mathscr{N}$ " stands for the replacement of $G$ by $2 G$. Neither $\mathcal{E}_{\mathscr{N}}^{\mathscr{B}}(3 ; M)$ nor $\mathcal{E}_{2, \mathcal{N}}^{\mathscr{B}}(2 ; M / 2)$ are known to be exactly computable, but the Helium-type ground state energy $\mathcal{E}_{2 . \mathcal{N}}^{\mathscr{B}}(2 ; M / 2)$ can certainly be computed in very accurate approximation by Hylleraas' variational method [Hyl30].

To state our second spin-off of Proposition 2 we recall that an upper bound to the bosonic ground state energy $\mathcal{E}_{\mathscr{N}, M}^{\mathscr{B}}(N)$ is obtained by estimating $Q_{V, M}^{(N)}\left(\psi^{(N)}\right)$ from above with a convenient trial wave function $\psi^{(N)} \equiv \phi^{\otimes N} \in$ $D_{Q}^{(N)}$, with $\phi \in \mathfrak{H}^{1}\left(\mathbb{R}^{3}\right)$. This gives $Q_{\mathcal{N}, M}^{(N)}\left(\phi^{\otimes N}\right)=\mathcal{H}_{\mathcal{K}, M}^{(N)}(\phi)$, where

$$
\mathcal{H}_{\mathcal{N}, M}^{(N)}(\phi)=N \frac{\hbar^{2}}{2 m} \mathcal{K}^{(1)}(\phi)-N G M m \mathcal{C}^{(1)}(\phi)-N(N-1) G m^{2} \frac{1}{2} I^{(2)}(\phi \otimes \phi)
$$

is a Hartree functional. Setting $\phi(\boldsymbol{q})=N^{3 / 2} \phi_{0}(N \boldsymbol{q})$, one easily obtains upper bounds on the Hamiltonian ground state energies which are $\propto-N^{3}(1+$ $O(1 / N))$. Pairing such an upper bound with Proposition 2 we obtain

Corollary 4. The limit $\lim _{N \uparrow \infty} N^{-3} \mathcal{E}_{\mathscr{N}}^{\mathscr{B}}(N ; M)$ exists and is non-trivial.

Our arguments do not reveal the nature of the limit, yet it is natural to conjecture that it is given by the minimum of the limiting Hartree functional

$$
\mathcal{H}_{\mathcal{N}}(\phi)=\frac{\hbar^{2}}{2 m} \mathcal{K}^{(1)}(\phi)-G m^{2} \frac{1}{2} I^{(2)}(\phi \otimes \phi)
$$

which does not feature $M$. This should be provable along the lines of [BeLi83] and [LiYa87]; see also [Lie90], [Thi02].

We stress that the above stated results hold for any grain's mass $M>0$.

\footnotetext{
${ }^{6}$ Incidentally, since all $\mathcal{E}_{\mathscr{N}}^{\mathscr{B}}\left(N ; 0^{+}\right)<0$, neither $\mathcal{E}_{\mathscr{N}}^{\mathscr{B}}(2 ; M)$ nor any $\mathcal{E}_{\mathscr{N}}^{\mathscr{B}}(N ; M)$ for $N>$ 2 can be estimated from below uniformly in $M$ by some fixed multiple of the explicitly computable one-body ground state energy $\mathcal{E}_{\mathscr{N}}^{\mathscr{B}}(1 ; M)=-\frac{1}{2} G^{2} M^{2} m^{3} / \hbar^{2}$.
} 


\subsection{Bosonic stars: the Galilei-invariant model}

When $M=0$ then the "min" in (13) has to be replaced by "inf." Yet, for $M=0$ the Hamiltonian (111) can be decomposed as

$$
H_{\mathscr{N}, 0}^{(N)} \equiv H_{\text {free }}^{(1)}+H_{\mathscr{N} \text {,int }}^{(N-1)},
$$

where $H_{\text {free }}^{(1)}$ is the Hamiltonian of a single non-interacting (free) particle of mass $N m$, describing the center-of-mass motion of the $N$-body system,

$$
H_{\text {free }}^{(1)} \equiv \frac{1}{2 N m}|\boldsymbol{P}|^{2},
$$

where $\boldsymbol{P}=\sum_{k=1}^{N} \boldsymbol{p}_{k}$, while $H_{\mathscr{N} \text {,int }}^{(N-1)}$ is the reduced Hamiltonian for the remaining degrees of freedom of the $N$-body system, describing the system-intrinsic motions, in effect an $N$-1-body problem. The intrinsic Hamiltonian is written most symmetrically in the vector variables of (11), viz.

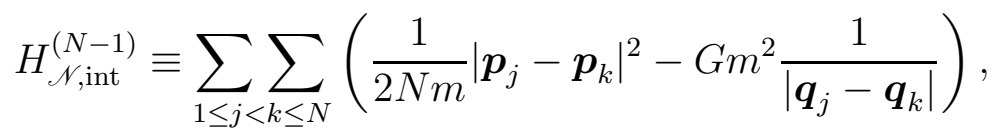

see the first (unnumbered) equation on p.382 in [HaPo67], see also Eq.(2.3) in Hal88; however, only $N-1$ vectors of the set $\left\{\boldsymbol{q}_{k}\right\}_{k=1}^{N}$ are linearly independent — in other words, the vectors in $\left\{\boldsymbol{q}_{k}\right\}_{k=1}^{N}$ are linear combinations of $N-1$ basis vectors in the linear subspace $\{\boldsymbol{Q} \equiv \mathbf{0}\}^{\perp} \subset \mathbb{R}^{3 N}$, where $\boldsymbol{Q}=N^{-1} \sum_{k=1}^{N} \boldsymbol{q}_{k}$ is the position vector of the system's center-of-mass canonically conjugate to $\boldsymbol{P}$ (up to scaling). Orthogonal transformations from $\left\{\boldsymbol{q}_{k}\right\}_{k=1}^{N}$ to $\{\boldsymbol{Q}\} \cup\left\{\overline{\boldsymbol{q}}_{k}\right\}_{k=1}^{N-1}$ having unit Jacobian determinant are described in [Pos56] and [Hal88], for instance. The Friedrichs extension of the intrinsic Hamiltonian (19) takes its minimum on its form domain $\mathfrak{H}^{1}\left(\mathbb{R}^{3}\right) \otimes \cdots \otimes \mathfrak{H}^{1}\left(\mathbb{R}^{3}\right) \subset \mathfrak{L}^{2}\left(\mathbb{R}^{3(N-1)}\right)$, and its minimum agrees with the infimum of the full Hamiltonian (11) for $M=0$.

Proposition 3. Let $\mathcal{E}_{\mathscr{N}}^{\mathscr{B}}(N ; 0)$ denote the bosonic ground state energies defined in (13) with $M=0$ and "min" replaced by "inf." Then for $N \geq 2$ the ratio $\mathcal{E}_{\mathscr{N}}^{\mathscr{B}}(N ; 0) / \mathcal{P}_{\mathscr{C}}(N)$ is finite and grows monotonically in $N$. Here, $\mathcal{P}_{\mathscr{C}}(N)=$ $N^{2}(N-1)$ is the same polynomial which occurs in the "atomic" Proposition 1 .

Corollary 5. For $N>1$ we have

$$
\mathcal{E}_{\mathscr{N}}^{\mathscr{B}}(N ; 0) \geq \mathcal{E}_{\mathscr{N}}^{\mathscr{B}}(2 ; 0) \frac{1}{4} N^{3}\left(1-N^{-1}\right) .
$$

The inequality (20) is sharp for $N=2$, but far from optimal when $N \gg 1$. The lower bound (20) is in fact known since [Pos62], where it was proved with different arguments; see also [Hal80], [Hal83], [BMR90]. A slightly weaker bound was obtained in [L-L69], where it was proved that for $N \geq 2$,

$$
\mathcal{E}_{\mathscr{N}}^{\mathscr{B}}(N ; 0) \geq \mathcal{E}_{\mathscr{N}}^{\mathscr{B}}(2 ; 0) \frac{1}{2} N^{3}\left(1-N^{-1}\right)^{2} .
$$


Also inequality (21) is sharp for $N=2$, but far from optimal when $N \gg 1$. We remark that one can explicitly compute $\mathcal{E}_{\mathscr{N}}^{\mathscr{B}}(2 ; 0)=-\frac{1}{4} G^{2} m^{5} / \hbar^{2}$.

Next we recall that in [Hal80] also the mirror-symmetric upper bound

$$
\mathcal{E}_{\mathscr{N}}^{\mathscr{B}}(N ; 0) \leq-B N^{3}\left(1-N^{-1}\right)
$$

was proved without invoking a Hartree functional (more explicitly, set $p=-1$ in formula (1.3) in Hal83]). Yet, while formula (1.3) in Hal83] does not imply that $\mathcal{E}_{N}^{\mathscr{B}}(N ; 0) / \mathcal{P}_{\mathscr{C}}(N)$ converges as $N \rightarrow \infty$, our Proposition 3 paired with Hall's upper bound (22) gives right away

Corollary 6. The limit $\lim _{N \uparrow \infty} \mathcal{E}_{\mathscr{N}}^{\mathscr{B}}(N ; 0) / \mathcal{P}_{\mathscr{C}}(N)$ exists and is non-trivial.

It is known Thi02 that the limit is given by the minimum of the Hartree functional (16). Of course, the upper bound $\mathcal{E}_{\mathscr{N}}^{\mathscr{B}}(N ; 0) \leq-C N^{3}$ with $C$ coming from Hartree theory, obtained earlier in [Pos62], [L-L69], can also be paired with Proposition 3 to yield Corollary 6 .

By the way, the convergence of the various monotonic increasing sequences in Propositions 1, 2, and 3 follows already from the negativity of all the ground state energies - what does not follow, then, is the nontriviality of the limits.

\section{Proofs}

Our Propositions 1, 2, and 3 are inspired by a monotonicity result for classical ground state energies proved in Kie09a and elaborated on in Kie09b. The classical proposition also covers Coulomb charges which, instead of being attracted by a nucleus, are confined to a sphere or some other compact domain, and then $\mathcal{E}_{\mathscr{C}}^{c l}(N) / \mathcal{P}_{\mathscr{C}}^{c l}(N)$ grows monotonically, where $\mathcal{E}_{\mathscr{C}}^{c l}(N)$ is the classical Coulomb ground state energy and $\mathcal{P}_{\mathscr{C}}^{c l}(N)=N(N-1)$. We shall rewrite the functionals of the quantum ground state energy variational principles into a quasi-classical format and then recycle the classical estimates. Yet the proofs of Propositions 1, 2, and 3 do not just consist of such variants of the classical estimate in [Kie09a, Kie09b] but also use the virial theorem in an essential way; the virial theorem plays no rôle in the classical proof. Incidentally, to apply the virial theorem we need $\mathcal{E}^{\mathscr{B}}(N)$ to be a minimum, not just an infimum.

\subsection{Proof of Proposition 1}

We begin by rewriting the quadratic form $Q_{\mathscr{C}}^{(N)}\left(\psi^{(N)}\right)$ into the convenient format of a quasi-classical expectation functional. Recall the physicists' nonunitary Fourier transform 7 of $\psi^{(N)}$,

$$
\widehat{\psi}_{\hbar}^{(N)}\left(\boldsymbol{p}_{1}, \ldots, \boldsymbol{p}_{N}\right):=\int \psi^{(N)}\left(\boldsymbol{q}_{1}, \ldots, \boldsymbol{q}_{N}\right) e^{-i \boldsymbol{p} \cdot \boldsymbol{q} / \hbar} d^{3 N} q,
$$

\footnotetext{
${ }^{7}$ This differs only by scaling from the conventional unitary Fourier transform.
} 
so that

$$
\psi^{(N)}\left(\boldsymbol{q}_{1}, \ldots, \boldsymbol{q}_{N}\right)=\frac{1}{h^{3 N}} \int \widehat{\psi}_{\hbar}^{(N)}\left(\boldsymbol{p}_{1}, \ldots, \boldsymbol{p}_{N}\right) e^{i \boldsymbol{p} \cdot \boldsymbol{q} / \hbar} d^{3 N} q
$$

where $h=2 \pi \hbar$ is Planck's quantum of action. This Fourier transform is a non-isometric isomorphism of $\mathfrak{L}^{2}\left(\mathbb{R}^{3 N}\right)$, so when $\left\|\psi^{(N)}\right\|_{\mathfrak{L}^{2}\left(\mathbb{R}^{3 N}\right)}=1$, then

$$
\int\left|\widehat{\psi}_{\hbar}^{(N)}\left(\boldsymbol{p}_{1}, \ldots, \boldsymbol{p}_{N}\right)\right|^{2} d^{3 N} p=h^{3 N}
$$

Clearly, $h^{-3 N}\left|\widehat{\psi}_{\hbar}^{(N)}\right|^{2}\left|\psi^{(N)}\right|^{2} \geq 0$, and $\iint h^{-3 N}\left|\widehat{\psi}_{\hbar}^{(N)}\right|^{2}\left|\psi^{(N)}\right|^{2} d^{3 N} p d^{3 N} q=1$ when $\left\|\psi^{(N)}\right\|_{\mathfrak{L}^{2}\left(\mathbb{R}^{3 N}\right)}=1$, so we can think of $h^{-3 N}\left|\widehat{\psi}_{\hbar}^{(N)}\right|^{2}\left|\psi^{(N)}\right|^{2}$ as a formal probability density function on the $N$-body phase space of points $\left(\boldsymbol{p}_{1}, \ldots, \boldsymbol{p}_{N} ; \boldsymbol{q}_{1}, \ldots, \boldsymbol{q}_{N}\right) \in$ $\mathbb{R}^{6 N}$. With the help of this Fourier transform, and integration by parts, we can rewrite the quadratic form (2) into a quasi-classical ensemble average thusly,

$$
Q_{\mathscr{C}}^{(N)}\left(\psi^{(N)}\right)=\iint H_{\mathscr{C}}^{(N)} h^{-3 N}\left|\widehat{\psi}_{\hbar}^{(N)}\right|^{2}\left|\psi^{(N)}\right|^{2} d^{3 N} p d^{3 N} q=:\left\langle H_{\mathscr{C}}^{(N)}\right\rangle_{\psi^{(N)}}
$$

where the double integral extends over $\mathbb{R}^{6 N}$, and $H_{\mathscr{C}}^{(N)}\left(\boldsymbol{p}_{1}, \ldots, \boldsymbol{q}_{N}\right)$ now is the classical Hamiltonian with Coulomb interactions, formally also given by (1) but now with $\boldsymbol{p}_{k} \in \mathbb{R}^{3}$; we use the same symbol for the Hamiltonian operator and its classical counterpart, as the context makes it unambiguously clear which object is meant.

Using next a familiar trick of Fisher and Ruelle [FiRu66] and Dyson and Lenard DyLe67, we rewrite the Coulomb Hamiltonian (1) as a double sum,

$$
H_{\mathscr{C}}^{(N)} \equiv \sum_{1 \leq k<l \leq N} \sum_{k, l} U_{k}^{(N)}
$$

where

$$
U_{k, l}^{(N)}:=\frac{1}{2 m(N-1)}\left(\left|\boldsymbol{p}_{k}\right|^{2}+\left|\boldsymbol{p}_{l}\right|^{2}\right)-\frac{N z^{2} e^{2}}{N-1}\left(\frac{1}{\left|\boldsymbol{q}_{k}\right|}+\frac{1}{\left|\boldsymbol{q}_{l}\right|}\right)+z^{2} e^{2} \frac{1}{\left|\boldsymbol{q}_{k}-\boldsymbol{q}_{j}\right|} .
$$

The superscript ${ }^{(N)}$ at $U_{k, l}^{(N)}$ reminds us of the explicit $N$ dependence exhibited at r.h.s.(28). With the help of (27) the quadratic form alias expectation functional (26) becomes

$$
Q_{\mathscr{C}}^{(N)}\left(\psi^{(N)}\right) \equiv \sum_{1 \leq k<l \leq N} \sum_{k, l}\left\langle U_{\psi^{(N)}}^{(N)} .\right.
$$

The double sum at r.h.s.(29) can be represented graph-theoretically as a complete $N$-graph with vertices numbered $1, \ldots, N$, with a value $\left\langle U_{k, l}^{(N)}\right\rangle_{\psi^{(N)}}$ assigned to the bond between the $k$-th and $l$-th vertex. An elementary graphtheoretical identity used in the classical proof in [Kie09a, Kie09b] says that 
such a sum over all bonds in a complete $N$-graph with $N>2$ equals $(N-2)^{-1}$ times the sum over all bonds of all its complete $N-1$-subgraphs. So for $N>2$,

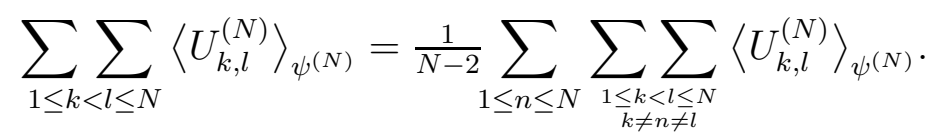

Note that (30) holds without any particular symmetry assumption on $\psi^{(N)}$.

We now start our estimates. Writing $\min _{\psi^{(N)}}$ for the minimum over the subset of $D_{Q}^{(N)}$ satisfying $\left\|\psi^{(N)}\right\|_{\mathfrak{L}^{2}\left(\mathbb{R}^{3 N}\right)}=1$, for $N>2$ we find

$$
\begin{aligned}
& \mathcal{E}_{\mathscr{C}}^{\mathscr{B}}(N)=\min _{\psi^{(N)}} \sum_{1 \leq k<l \leq N} \sum_{k, l}\left\langle U_{k, l}^{(N)}\right\rangle_{\psi^{(N)}} \\
& \geq \frac{1}{N-2} \sum_{1 \leq n \leq N} \min _{\psi^{(N)}} \sum_{\substack{1 \leq k<l \leq N \\
k \neq n \neq l}} \sum_{k, l}\left\langle U_{k, 1}^{(N)}\right\rangle_{\psi^{(N)}} \\
& \geq \frac{1}{N-2} \sum_{1 \leq n \leq N} \min _{\psi^{(N-1)}} \sum_{\substack{1 \leq k<l \leq N \\
k \neq n \neq l}} \sum_{k, l}\left\langle U_{k(N)}^{(N)}\right\rangle_{\psi^{(N-1)}}
\end{aligned}
$$

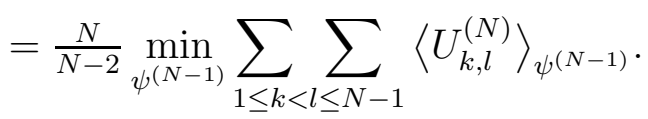

The first equality in (31) is just definition (6) and identity (29). For the first inequality in (31) we used identity (30) and the fact that the minimum of a sum is never lesser than the sum of the minima; actually, this inequality is in general strict. For the second inequality in (31) we used that the to-be-minimized double sums before that " $\geq$ " symbol each involve only expectations computed with an $N-1$ point marginal of $h^{-3 N}\left|\widehat{\psi}_{\hbar}^{(N)}\right|^{2}\left|\psi^{(N)}\right|^{2}$, which can be written as averages of conditional expectations - conditioning is on the $\left(\boldsymbol{p}_{n}, \boldsymbol{q}_{n}\right)$ variables of $\psi^{(N)}$ - and the inequality results when the conditioning is relaxed; incidentally, since we do not impose any symmetry on the various $\psi$, the inequality symbol " $\geq$ " can actually be replaced by the equality sign "=" (just tensor multiply each $N-1$ point minimizing wave function with any nice 1 point wave function in the respective $n$-th variables), but if bosonic (or fermionic) symmetry is imposed, then the " $\geq$ " is generally even a " $>$." For the final equality we used the permutation symmetry of $U_{k, l}^{(N)}$.

Recalling (27) and (28), and letting $\widetilde{\psi}_{\mathscr{B}}^{(N-1)}$ denote the normalized minimizer (which exists!) of $\sum \sum\left\langle U_{k, l}^{(N)}\right\rangle_{\psi^{(N-1)}}$, with the double sum running over $1 \leq k<l \leq N-1$, the last expression in (31) can be recast as follows,

$$
\begin{aligned}
\min _{\psi^{(N-1)}} \sum_{1 \leq k<l \leq N-1} \sum_{k, l}\left\langle U_{\psi^{(N-1)}}^{(N)}=\left\langle H_{\mathscr{C}}^{(N-1)}\right\rangle_{\widetilde{\psi}_{\mathscr{B}}^{(N-1)}}-\right. \\
\frac{1}{(N-1)(N-2)} \sum_{1 \leq k<l \leq N-1} \sum_{1 \leq k}\left\langle H_{\mathscr{C}, k}^{(1)}+H_{\mathscr{C}, l}^{(1)}\right\rangle_{\widetilde{\psi}_{\mathscr{B}}^{(N-1)}},
\end{aligned}
$$


where

$$
H_{\mathscr{C}}^{(1)}:=\frac{1}{2 m}|\boldsymbol{p}|^{2}-z^{2} e^{2} \frac{1}{|\boldsymbol{q}|}
$$

is a familiar Hydrogen-type Hamiltonian, and $H_{\mathscr{C}, k}^{(1)}$ and $H_{\mathscr{C}, l}^{(1)}$ indicate that (33) is expressed in the $k$-th and $l$-th particle's variables, respectively. To handle the Hydrogen-like contributions in the last line of (31) we use the virial theorem, which for $N>2$ furnishes the identity

$$
\begin{aligned}
&\left.-\frac{1}{N-2} \sum_{1 \leq k<l \leq N-1} \sum_{1 \leq H_{\mathscr{C}, k}}+H_{\mathscr{C}, l}^{(1)}\right\rangle_{\widetilde{\psi}_{\mathscr{B}}^{(N-1)}}= \\
&\left\langle H_{\mathscr{C}}^{(N-1)}\right\rangle_{\widetilde{\psi}_{\mathscr{B}}^{(N-1)}}+\frac{1}{N-2} z^{2} e^{2} I^{(N-1)}\left(\widetilde{\psi}_{\mathscr{B}}^{(N-1)}\right),
\end{aligned}
$$

and since $I^{(N-1)}\left(\widetilde{\psi}_{\mathscr{B}}^{(N-1)}\right)>0$, we obtain the estimate

$$
\left.-\frac{1}{N-2} \sum_{1 \leq k<l \leq N-1} \sum_{\langle}\left\langle H_{\mathscr{C}, k}^{(1)}+H_{\mathscr{C}, l}^{(1)}\right\rangle_{\widetilde{\psi}_{\mathscr{B}}^{(N-1)}}\right\rangle\left\langle H_{\mathscr{C}}^{(N-1)}\right\rangle_{\widetilde{\psi}_{\mathscr{B}}^{(N-1)}} .
$$

Estimates (31) together with identities (32) and (34) and the estimate (35), plus an obvious inequality, now give, for $N>2$,

$$
\begin{aligned}
\mathcal{E}_{\mathscr{C}}^{\mathscr{B}}(N) & >\frac{N^{2}}{(N-1)(N-2)}\left\langle H_{\mathscr{C}}^{(N-1)}\right\rangle_{\widetilde{\psi}_{\mathscr{B}}^{(N-1)}} \\
& \geq \frac{N^{2}}{(N-1)(N-2)} \min _{\psi^{(N-1)}}\left\langle H_{\mathscr{C}}^{(N-1)}\right\rangle_{\psi^{(N-1)}} \\
& =\frac{N^{2}}{(N-1)(N-2)} \mathcal{E}_{\mathscr{C}}^{\mathscr{B}}(N-1) .
\end{aligned}
$$

Finally, dividing (36) by $N^{2}(N-1)$ yields, for $N>2$,

$$
\frac{1}{N^{2}(N-1)} \mathcal{E}_{\mathscr{C}}^{\mathscr{B}}(N)>\frac{1}{(N-1)^{2}(N-2)} \mathcal{E}_{\mathscr{C}}^{\mathscr{B}}(N-1)
$$

and the proof of the monotonic increase of the map $N \mapsto \mathcal{E}_{\mathscr{C}}^{\mathscr{B}}(N) / \mathcal{P}_{\mathscr{C}}(N)$, defined for $N \geq 2$, is complete.

\subsection{Proof of Proposition 2}

Up to where the virial identity is needed the proof of Proposition 2 follows verbatim the proof of Proposition 1. The different order-three polynomials in Propositions 1 and 2 are the result of necessarily different "end games."

Thus, we first rewrite the quadratic form $Q_{\mathcal{N}, M}^{(N)}\left(\psi^{(N)}\right)$ into the more convenient format of a quasi-classical expectation functional,

$$
Q_{\mathscr{N}, M}^{(N)}\left(\psi^{(N)}\right)=\iint H_{\mathscr{N}, M}^{(N)} h^{-3 N}\left|\widehat{\psi}_{\hbar}^{(N)}\right|^{2}\left|\psi^{(N)}\right|^{2} d^{3 N} p d^{3 N} q=:\left\langle H_{\mathscr{N}, M}^{(N)}\right\rangle_{\psi^{(N)}}
$$


where $H_{\mathscr{N}, M}^{(N)}\left(\boldsymbol{p}_{1}, \ldots, \boldsymbol{q}_{N}\right)$ is again the classical Hamiltonian with Newton interactions, formally also given by (11) but now with $\boldsymbol{p}_{k} \in \mathbb{R}^{3}$. Once again following Fisher and Ruelle [FiRu66], Dyson and Lenard [DyLe67], and LèvyLeblond [L-L69], we rewrite the Newton Hamiltonian (11) as a double sum,

but now

$$
H_{\mathscr{N}, M}^{(N)} \equiv \sum_{1 \leq k<l \leq N} \sum_{k, l} U^{(N)}
$$

$$
U_{k, l}^{(N)}:=\frac{1}{2 m(N-1)}\left(\left|\boldsymbol{p}_{k}\right|^{2}+\left|\boldsymbol{p}_{l}\right|^{2}\right)-\frac{G M m}{N-1}\left(\frac{1}{\left|\boldsymbol{q}_{k}\right|}+\frac{1}{\left|\boldsymbol{q}_{l}\right|}\right)-G m^{2} \frac{1}{\left|\boldsymbol{q}_{k}-\boldsymbol{q}_{j}\right|} .
$$

The ${ }^{(N)}$ at $U_{k, l}^{(N)}$ reminds us of the explicit $N$ dependence at r.h.s.(40). With (39) the quadratic form alias expectation functional (38) becomes

$$
Q_{V, M}^{(N)}\left(\psi^{(N)}\right) \equiv \sum_{1 \leq k<l \leq N} \sum_{k, l}\left\langle U_{\psi^{(N)}}^{(N)}\right.
$$

and as before, for $N>2$ we have the identity

$$
\sum_{1 \leq k<l \leq N} \sum_{\langle}\left\langle U_{k, l}^{(N)}\right\rangle_{\psi^{(N)}}=\frac{1}{N-2} \sum_{1 \leq n \leq N} \sum_{\substack{1 \leq k<l \leq N \\ k \neq n \neq l}} \sum_{\substack{1 \\ k, l}}\left\langle U_{\psi^{(N)}}^{(N)} .\right.
$$

Note that (42) holds without assuming any particular symmetry of $\psi^{(N)}$.

We now start our estimates. Writing $\min _{\psi^{(N)}}$ for the minimum over the subset of $D_{Q}^{(N)}$ satisfying $\left\|\psi^{(N)}\right\|_{\mathfrak{L}^{2}\left(\mathbb{R}^{3 N}\right)}=1$, for $N>2$ we find

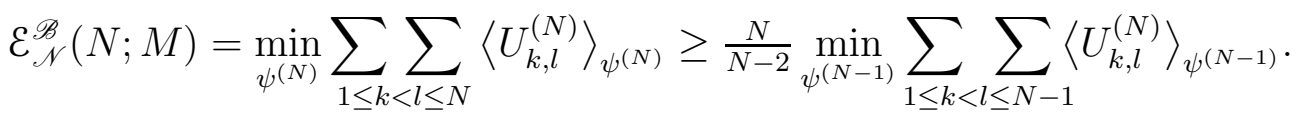

All the steps to get (43) are identical to the corresponding steps which yield formula (31).

The last expression can be recast with the help of elementary algebra, thus

$$
\begin{aligned}
\min _{\psi^{(N-1)}} \sum_{1 \leq k<l \leq N-1} \sum_{l \leq N}\left\langle U_{k, l}^{(N)}\right\rangle_{\psi^{(N-1)}}=\langle & \left.H_{\mathscr{N}, M}^{(N-1)}\right\rangle_{\widetilde{\psi}_{\mathscr{B}, M}^{(N-1)}}- \\
& \frac{1}{(N-1)(N-2)} \sum_{1 \leq k<l \leq N-1} \sum_{\mathscr{N}, M}\left\langle H_{\mathscr{N}}^{(1, k)}+H_{\mathscr{N}, M}^{(1, l)}\right\rangle_{\widetilde{\psi}_{\mathscr{B}, M}^{(N-1)}},
\end{aligned}
$$

where $\widetilde{\psi}_{\mathscr{B}, M}^{(N-1)}$ denotes the normalized minimizer of $\sum \sum\left\langle U_{k, l}^{(N)}\right\rangle_{\psi^{(N-1)}}$, the double sum running over $1 \leq k<l \leq N-1$, and where

$$
H_{\mathscr{N}, M}^{(1)}:=\frac{1}{2 m}|\boldsymbol{p}|^{2}-G M m \frac{1}{|\boldsymbol{q}|}
$$


is a familiar Hydrogen-type Hamiltonian, and the superscripts ${ }^{k}$ and ${ }^{l}$ at $H_{\mathscr{N}, M}^{(1, \cdot)}$ indicate that (45) is expressed in the $k$-th and $l$-th particle's variables, respectively. Curiously, the identity (44) agrees exactly with the one in (32); naively one might have expected that the difference in the $N$-dependence of the central terms, viz. $N z^{2} e^{2}$ vs. $G M m$, would already show itself at this point, but it does not. Be that as it may, the strict similarity between the proofs of Propositions 1 and 2 ends here.

Namely, while we will also use the virial theorem to handle the Hydrogenlike terms in the last line of (43), we now first recast these terms as follows,

$$
\begin{aligned}
\frac{1}{N-2} \sum_{1 \leq k<l \leq N-1} \sum_{l}\left\langle H_{\mathscr{N}, M}^{(1, k)}+H_{\mathscr{N}, M}^{(1, l)}\right\rangle_{\widetilde{\psi}_{\mathscr{B}, M}^{(N-1)}}= \\
\frac{\hbar^{2}}{2 m} \mathcal{K}^{(N-1)}\left(\widetilde{\psi}_{\mathscr{B}, M}^{(N-1)}\right)-G M m C^{(N-1)}\left(\widetilde{\psi}_{\mathscr{B}, M}^{(N-1)}\right) .
\end{aligned}
$$

For $N>3$ the virial theorem now yields the identity

$\frac{\hbar^{2}}{2 m} \mathcal{K}^{(N-1)}\left(\widetilde{\psi}_{\mathscr{B}, M}^{(N-1)}\right)=-\frac{N-1}{N-3}\left\langle H_{\mathscr{N}, M}^{(N-1)}\right\rangle_{\widetilde{\psi}_{\mathscr{B}, M}^{(N-1)}}+\frac{1}{N-3} G M m C^{(N-1)}\left(\widetilde{\psi}_{\mathscr{B}, M}^{(N-1)}\right)$.

Estimates (43) together with the identities (46) and (47), plus the inequality $C^{(N-1)}\left(\widetilde{\psi}_{\mathscr{B}, M}^{(N-1)}\right)>0$, now give, for $N>3$,

$$
\begin{aligned}
\mathcal{E}_{\mathscr{N}}^{\mathscr{B}}(N ; M) & \geq \frac{N}{(N-3)}\left\langle H_{\mathscr{N}}^{(N-1)}\right\rangle_{\widetilde{\psi}_{\mathscr{B}, M}^{(N-1)}} \\
& \geq \frac{N}{(N-3)} \min _{\psi^{(N-1)}}\left\langle H_{\mathscr{N}}^{(N-1)}\right\rangle_{\psi^{(N-1)}} \\
& =\frac{N}{(N-3)} \mathcal{E}_{\mathscr{N}}^{\mathscr{B}}(N-1 ; M) ;
\end{aligned}
$$

the first inequality is strict if $N>4$. Dividing (48) by $N(N-1)(N-2)$ yields

$$
\frac{1}{N(N-1)(N-2)} \mathcal{E}_{\mathscr{N}}^{\mathscr{B}}(N ; M) \geq \frac{1}{(N-1)(N-2)(N-3)} \mathcal{E}_{\mathscr{N}}^{\mathscr{B}}(N-1 ; M)
$$

for $N>3$, with strict inequality for $N>4$. The proof of the monotonic increase of the map $N \mapsto \mathcal{E}_{\mathscr{N}}^{\mathscr{B}}(N ; M) / \mathcal{P}_{\mathscr{N}}(N)$, defined for $N \geq 3$, is complete.

\subsection{Proof of Proposition 3}

By the decomposition (17), the infimum of $\left\langle H_{\mathscr{N}, 0}^{(N)}\right\rangle_{\psi^{N}}$ equals the infimum of $\left\langle H_{\mathscr{N}, \text { int }}^{(N-1)}\right\rangle_{\bar{\psi}^{(N-1)}}$, which with the help of (19) can be shown to be a minimum; here, the overbar on $\bar{\psi}^{(N-1)}$ indicates dependence on $\left\{\overline{\boldsymbol{q}}_{k}\right\}_{k=1}^{N-1}$ only.

Writing $W_{k, l}^{(N)}$ for the summands on r.h.s.(19), we can apply our strategy of proof of Propositions 1 and 2, We just need to substitute $W_{k, l}^{(N)}$ for $U_{k, l}^{(N)}$ and $\bar{\psi}^{(N-1)}$ for $\psi^{N}$, respectively $\bar{\psi}^{(N-2)}$ for $\psi^{(N-1)}$, in (42) and (43) (with $M=0$ ), rewrite as in (44), with $\left\langle W_{k, l}^{(N)}\right\rangle_{\widetilde{\psi}_{\mathscr{B}}^{(N-2)}}$ in place of $\left\langle U_{k, l}^{(N)}\right\rangle_{\widetilde{\psi}_{\mathscr{B}, M}^{(N-1)}}$, and find

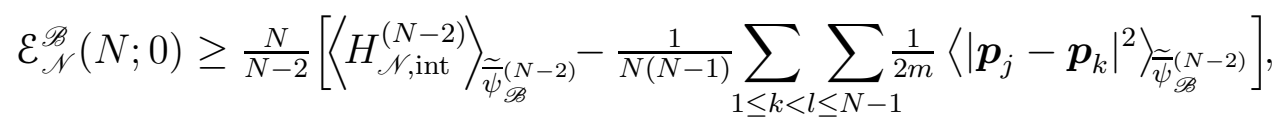


where $\widetilde{\bar{\psi}}_{\mathscr{B}}^{(N-2)}$ now denotes the normalized minimizer of $\sum \sum\left\langle W_{k, l}^{(N)}\right\rangle_{\bar{\psi}^{(N-2)}}$, the double sum running over $1 \leq k<l \leq N-1$. At this point the virial theorem enters once again, but in contrast to the proofs of Propositions 1 and 2 it here allows us to express r.h.s.(50) entirely in terms of the expectation value of the intrinsic Hamiltonian, without producing any additional terms which would have to be estimated. So at the end of the day, our proof yields, for $N>2$,

$$
\mathcal{E}_{\mathscr{N}}^{\mathscr{B}}(N ; 0) \geq \frac{N^{2}}{(N-1)(N-2)} \mathcal{E}_{\mathscr{N}}^{\mathscr{B}}(N-1 ; 0),
$$

and dividing (51) by $N^{2}(N-1)$ yields, for $N>2$,

$$
\frac{1}{N^{2}(N-1)} \mathcal{E}_{\mathscr{N}}^{\mathscr{B}}(N ; 0) \geq \frac{1}{(N-1)^{2}(N-2)} \mathcal{E}_{\mathscr{N}}^{\mathscr{B}}(N-1 ; 0) .
$$

The proof of the monotonic increase of the map $N \mapsto \mathcal{E}_{\mathscr{N}}^{\mathscr{B}}(N ; 0) / \mathcal{P}_{\mathscr{C}}(N)$, defined for $N \geq 2$, is complete.

\section{Further spin-offs: Hall-Post inequalities}

Our Propositions 1, 2, and 3 are each equivalent to a statement that the $N$ body ground state energy $\mathcal{E}(N)$ is bounded below by a specific $N$-dependent multiple of the $N-1$-body ground state energy $\mathcal{E}(N-1)$; more precisely, when $N>N_{0}$ (with $N_{0}=2$ or 3 ) then $\mathcal{E}(N) \geq R(N) \mathcal{E}(N-1)$, where $R(N)$ is some rational function of $N$. Here the so-compared $N$-body and $N-1$-body Hamiltonians (11), respectively (19), of our bosonic gravitational systems differ only in their number of particles, and the Hamiltonians (11) of the bosonic atoms differ only in the number of their "bosonic electrons" and the corresponding charge of the atomic nucleus. Within the adapted approximations (neglecting: relativity, spin degrees of freedom, nuclear motion, etc.) our Propositions are therefore statements about sequences of systems as nature would supply them. These monotonicity results seem not to have been known before.

Inspection of our proofs of Propositions 1, 2, and 3 reveals that these proofs establish also technically somewhat stronger lower bounds for $\mathcal{E}(N)$ in terms of a specific $N$-dependent multiple of the $N$-1-body ground state energy $\mathcal{E}^{\prime}(N-1)$ of an $N-1$-body system with suitably rescaled coupling constants. Such inequalities are known as Hall-Post inequalities; cf. [KhRi01. Indeed, our inequalities (31), (43) and (50) in this paper are identical, in essence if not in appearance, to Hall-Post inequalities for our Hamiltonians. Since the masses and charges of the various "elementary" particles of nature cannot be rescaled, nor can the "constants of nature," these intermediate inequalities ("intermediate" regarding proving our propositions) are therefore generally not statements about sequences of systems which nature could supply.

There is (at least) one possible exception, though, to what we just wrote. Namely, in a certain quantum-mechanical approximation to QCD in which a 
baryon is made of an $\mathrm{N}$-quark color-singlet state ART82 the Hamiltonian has a factor $1 /(N-1)$ in front of the pair interaction potential, and in this case the Hall-Post inequality relating the $N$ - and the $N$ - 1-body systems is precisely an inequality between the ground state energies of the $N$-quark and the $N-1$-quark Hamiltonians. So in this case the Hall-Post inequality itself produces a monotonicity result for "physical" (i.e., according to that model) baryon masses $M(N)$, viz. the sequence $N \mapsto M(N) / N$ is monotonic increasing - see (2.7) in ART82. The proof of (2.7) in ART82 would not satisfy a mathematician, but it is "morally correct" and can easily be made rigorous (for a large class of pair-interaction potentials). Our strategy of proof is rigorous and produces the monotonicity result of ART82 in "step one" (NB: the virial theorem is not needed with $1 /(N-1)$-rescaled pair interactions). Incidentally, the result of [ART82] is meant for fermionic quarks, but it holds for Bosons as well (see also our concluding remarks in the next section).

The monotonicity result of ART82 has spin-offs analogous to our corollaries, not noted in [ART82]. Thus, for "bosonic quarks" an upper bound on the quark-specific baryon mass $M(N) / N$ as defined by the approximation to QCD of ART82 follows easily from the Hartree approximation, which together with the monotonic increase implies that the sequence $N \mapsto M(N) / N$ converges to a nontrivial limit. For fermionic quarks $N \mapsto M(N) / N$ is unbounded.

Hall-Post inequalities between the ground state energies of $N$-body and $N-K$-body systems with $K=1,2, \ldots, N-2$ and appropriately rescaled coupling constants were first established in Hal80, picking up on earlier work in [Pos56], [HaPo67] where $K=N-2$. However, the gist of the Hall-Post type proofs of the so-named inequalities is quite different from ours and relies heavily on the symmetry (or antisymmetry) of the wave function, which implies (in self-explanatory notation) that for each $N$ and each $\psi^{(N)}$ all the $\left\langle|\boldsymbol{p}|_{k}^{2}\right\rangle_{\psi^{(N)}}$ have a common value, all the $\left\langle V_{k, l}\right\rangle_{\psi^{(N)}}$ have a common value, etc. By contrast, our proof of inequalities (31), (43), and (50) in this paper does not make use of any symmetry of the wave functions and works equally well when the minimization is carried out over some subset of completely unsymmetric wave functions, should the demand arise. Moreover, with wave functions replaced by classical configurations, our technique handles also the classical ground state problems with pair interactions $V\left(\boldsymbol{q}_{k}, \boldsymbol{q}_{l}\right)$ which are bounded below. Typically the $V\left(\boldsymbol{q}_{k}, \boldsymbol{q}_{l}\right)$ in a classical ground state configuration have no common value, so that the Hall-Post strategy would fail to prove Proposition 1 in [Kie09b.

To summarize, our proofs can be characterized in a nutshell by saying that their basic ingredients are the Hall-Post inequality and the virial identity for the respective Hamiltonian under study, plus some obvious positivity inequality - except that we did not start from any Hall-Post inequality but instead obtained the relevant inequality from scratch with a more flexible type of proof which does not utilize any symmetry of the wave functions. 


\section{Concluding remarks}

Our Propositions 1, 2, and 3are statements about the bosonic ground state energies of atoms and stars. However, since we have not used any particular symmetry of $\psi^{(N)}$ in our estimates, Propositions 1, 2, and 3 and their proofs hold verbatim also for fermionic ground state energies $\mathcal{E}_{\mathscr{C}}^{\mathscr{F}}(N)$ and $\mathcal{E}_{\mathscr{N}}^{\mathscr{F}}(N ; M)$, with $M \geq 0$, obtained by minimizing only over the anti-symmetric subspace of $D_{Q}^{(N)}$. So we conclude that also $N \mapsto \mathcal{E}_{\mathscr{C}}^{\mathscr{F}}(N) / \mathcal{P}_{\mathscr{C}}(N)$ and $N \mapsto \mathcal{E}_{\mathscr{N}}^{\mathscr{F}}(N ; M) / \mathcal{P}_{\mathscr{N}}(N)$ and also $N \mapsto \mathcal{E}_{\mathscr{N}}^{\mathscr{F}}(N ; 0) / \mathcal{P}_{\mathscr{C}}(N)$ are monotonic increasing. Alas, Propositions 1. 2, and [3] are considerably less interesting for the ground state energies of fermionic atoms and stars than for their bosonic counterparts.

Indeed, since $\mathcal{E}_{\mathscr{C}}^{\mathscr{F}}(N) \asymp-C N^{7 / 3}$ for large $N$ [Lie90], Thi02], the monotonicity of $N \mapsto \mathcal{E}_{\mathscr{C}}^{\mathscr{F}}(N) / \mathcal{P}_{\mathscr{C}}(N)$ is far from optimal. An optimal polynomial monotonicity result for the ground state energies of fermionic atoms would state that $N \mapsto \mathcal{E}_{\mathscr{C}}^{\mathscr{F}}(N) / \mathcal{P}_{\mathscr{C}}^{\mathscr{F}}\left(N^{1 / 3}\right)$ is monotonic increasing, where $\mathcal{P}_{\mathscr{C}}^{\mathscr{F}}(\cdot)$ is a polynomial of degree 7 . In comparison, by the upper bound $\mathcal{E}_{\mathscr{C}}^{\mathscr{B}}(N) \leq$ $-C N^{3}$ from Hartree theory one cannot improve our monotonicity result for the bosonic atomic ground state energies to any lower leading power in $N$.

The same remarks apply mutatis mutandis also to stars. Namely, since $\mathcal{E}_{\mathscr{N}}^{\mathscr{F}}(N ; 0) \asymp-C N^{7 / 3}$ for large $N$ (see [Lan38] for a formal argument and Thi02 for a proof), and presumably also $\mathcal{E}_{\mathscr{N}}^{\mathscr{F}}(N ; M) \asymp-C N^{7 / 3}$ for large $N$, the monotonicity of $N \mapsto \mathcal{E}_{\mathscr{N}}^{\mathscr{F}}(N ; M) / \mathcal{P}_{\mathscr{N}}(N)$ and of $N \mapsto \mathcal{E}_{\mathscr{N}}^{\mathscr{F}}(N ; 0) / \mathcal{P}_{\mathscr{C}}(N)$ is far from optimal polynomial monotonicity for fermionic stars, namely that $N \mapsto \mathcal{E}_{\mathscr{N}}^{\mathscr{F}}(N ; M) / \mathcal{P}_{\mathscr{N}, M}^{\mathscr{F}}\left(N^{1 / 3}\right)$ is monotonic increasing, where $\mathcal{P}_{\mathscr{N}, M}^{\mathscr{F}}(\cdot)$ is a polynomial of degree 7 , indexed by $M \geq 0$. On the other hand, one cannot improve our monotonicity result for the bosonic stellar ground state energies to any smaller leading power in $N$, for $\mathcal{E}_{\mathscr{N}}^{\mathscr{B}}(N ; M) \leq-C N^{3}$ by Hartree theory.

To prove the optimal polynomial monotonicity for the fermionic ground state energies of atoms and stars, if possible at all, will require detailed input about the structure of the antisymmetric subspace of the form domain. Yet it may be hoped that the techniques developed in this paper will serve as an important stepping stone towards such fermionic proofs.

Acknowledgment. This paper was written with support from the NSF under grant DMS-0807705. Any opinions expressed in this paper are entirely those of the author and not those of the NSF. I thank Elliott H. Lieb for drawing my attention to the work of Post and collaborators, and Detlev Buchholz, Jerry Percus, Walter Thirring, and two anonymous referees for their helpful comments. 


\section{References}

[ART82] Ader, J.-P., Richard, J.-M., and Taxil, P., "Do narrow heavy multiquark states exist?," Phys. Rev. D25:2370-2382 (1982).

[Aetal96] Appelquist, G., et al., "Antinuclei production in $\mathrm{Pb}+\mathrm{Pb}$ collisions at 158 A GeV/c," Phys. Lett. B376:245-250 (1996).

[Aetal03a] Arsenescu, R., et al., "Antihelium-3 production in lead-lead collisions at 158 A GeV/c," New J. Phys. 5:1.1-1.12 (2003).

[Aetal03b] Arsenescu, R., et al., "An investigation of the antinuclei and nuclei production mechanism in $\mathrm{Pb}+\mathrm{Pb}$ collisions at 158 A GeV," New J. Phys. 5:150.1-150.23 (2003).

[Bac91] Bach, V., "Ionization energies of bosonic Coulomb systems," Lett. Math. Phys. 21:139-149 (1991).

[BLLS93] Bach, V., Lewis, R., Lieb, E.H., and Siedentop, H., "On the number of bound states of a bosonic $N$-particle Coulomb system," Math. Z. 214:441-460 (1993).

[BMR90] Basdevant, J.L., Martin, A., and Richard, J.M. "Improved bounds on many-body Hamiltonians: I. Self-gravitating Bosons," Nucl. Phys. B343:60-68 (1990).

[BaSe01] Baumgartner B., and Seiringer, R. "Atoms with bosonic "electrons" in strong magnetic fields," Annales de l'I.H.P. 2:41-76 (2001).

[BeLi83] Benguria, R., and Lieb, E.H., "Proof of stability of highly negative ions in the absence of the Pauli principle," Phys. Rev. Lett. 50:1771-1774 (1983).

[CzKa00] Czarnecki, A., and Karshenboim, S.G. "Decays of Positronium," pp. 538-544 in: Proc. of the 14th International Workshop on High Energy Physics and Quantum Field Theory (QFTHEP99, Moscow 1999), B.B. Levchenko and V.I. Savrin (eds.), MSU-Press (2000).

[Des60] Desai, B.P., "Proton-antiproton annihilation in protonium," Phys. Rev. 119, 1385-1389 (1960).

[DyLe67] Dyson, F.J., and Lenard, A., "Stability of matter. I," J. Math. Phys. 8:423-434 (1967).

[FiRu66] Fisher, M.E., and Ruelle, D., "Stability of many-particle systems," J. Math. Phys. 7:260-270 (1966).

[FrLe09] Frank, R., and Lenzmann, E. "Uniqueness of ground states for the $\mathfrak{L}^{2}$ critical Boson star equation," arXiv:0905.3105 (2009).

[Hal80] Hall, R.L., "Energy trajectories for the $N$-boson problem by the method of potential envelopes," Phys. Rev. D 22:2062-2072 (1980).

[Hal83] Hall, R.L., "Energy inequalities $\left(\begin{array}{c}N \\ 2\end{array}\right)^{-1} E_{N} \leq\left(\begin{array}{c}K \\ 2\end{array}\right)^{-1} E_{K}, 2 \leq K<N$ relating two systems of identical bosons," Phys. Rev. D 27:2379-2382 (1983).

[Hal88] Hall, R.L., "The ground-state energy of a system of identical bosons," $J$. Math. Phys. 29:990-995 (1988). 
[Hal92] Hall, R.L., "Gravitating boson systems," Phys. Rev. A 45:7682-7687 (1992).

[HaLu06] Hall, R.L., and Lucha, W., "Gravitating semirelativistic N-boson systems," J. Phys. A 39:11531-11540 (2006).

[HaLu08] Hall, R.L., and Lucha, W., "Semirelativistic stability of $N$-boson systems bound by $1 / r_{i j}$ pair potentials," J. Phys. A 41:1751-8121 (2008).

[HaPo67] Hall, R.L., and Post, H.R., "Many-particle systems: IV. Short-range interactions," Proc. Phys. Soc. 90:381-396 (1967).

[Hyl30] Hylleraas, E.A. "Über den Grundterm der Zweielektronenprobleme von $H^{-}, H e, \mathrm{Li}^{+}, B e^{++}$, usw.," Z. Phys. 65:209-225 (1930).

[KhRi01] Khare, A., and Richard, J.-M., "Testing Hall-Post inequalities with exatly solvable N-body problems," J. Phys. A34:L447-L452 (2001).

[Kie09a] Kiessling, M.K.-H., "The Vlasov continuum limit for the classical microcanonical ensemble," Rev. Math. Phys., 21:1145-1195 (2009).

[Kie09b] Kiessling, M.K.-H., "A note on classical ground state energies," J. Stat. Phys., 136:275-284 (2009).

[KSK04] Kolomeisky, E.B., Straley, J.P., and Kalas, R.M., "Ground-state properties of artificial bosonic atoms, Bose interaction blockade, and the singleatom pipette," Phys. Rev. A 69:063401-17 (2004).

[Lan38] Landau, L.D., "Origin of stellar energy," Nature, 141: 333-334 (1938).

[L-L69] Lèvy-Leblond, J.-M., "Nonsaturation of gravitational forces", J. Math. Phys. 10:806-812 (1969).

[Lie90] Lieb, E. H., "The stability of matter: from atoms to stars," Bull. Amer. Math. Soc. 22, 1-49 (1990).

[LiYa87] Lieb, E.H., and Yau, H.-T., "The Chandrasekhar theory of stellar collapse as the limit of quantum mechanics," Commun. Math. Phys. 112:147-174 (1987).

[Pos56] Post, H.R., "Many-particle systems: II," Proc. Phys. Soc. A 69:936-938 (1956).

[Pos62] Post, H.R., "Many-particle systems: III. Determination of the ground state energy of a system of $N$ particles interacting by attractive inverse square forces," Proc. Phys. Soc. 79:819-820 (1962).

[ReSi78] Reed, M., and Simon, B., "Analysis of operators," Methods of modern mathematical physics IV, Academic Press, Orlando (1978).

[Rus97] Ruskai, M.B., "Improved estimate on the number of bound states of negatively charged bosonic atoms," Annales de l'I.H.P. 61:153-162 (1994).

[Sol90] Solovej, J.P. "Asymptotics for bosonic atoms," Lett. Math. Phys. 20:165172 (1990).

[Thi02] Thirring, W.E. "Quantum mathematical physics. Atoms, molecules, and large systems," second English ed., E.M. Harrell (transl.) Springer, New York (2002).

[Zetal06a] Zurlo, N., et al., "Evidence for the production of slow antiprotonic Hydrogen in vacuum," Phys. Rev. Lett. 97:153401-5 (2006).

[Zetal06b] Zurlo, N., et al., "Production of slow protonium in vacuum," Hyperfine Interactions 172:97-105 (2006). 